\title{
Near-infrared spectroscopy of Cepheids in the Galactic nuclear disk
}

\author{
Noriyuki Matsunaga ${ }^{1}$, Kei Fukue ${ }^{1}$, Naoto Kobayashi ${ }^{2}$, Yuji Ikeda ${ }^{3}$, \\ Ryo Yamamoto ${ }^{2}$, Shiori Kyu ${ }^{2}$, Satoshi Hamano ${ }^{2}$, Chikako Yasui ${ }^{1}$, \\ Takuji Tsujimoto ${ }^{4}$, Wako Aoki ${ }^{4}$, Shogo Nishiyama ${ }^{4}$, Tetsuya Nagata ${ }^{5}$, \\ Katia Genovali ${ }^{6}$, Laura Inno ${ }^{6}$ and Giuseppe Bono ${ }^{6}$ \\ ${ }^{1}$ Dept. Astronomy, The Univ. Tokyo, 7-3-1 Hongo, Bunkyo, Tokyo 113-0033, Japan \\ ${ }^{2}$ Institute of Astronomy, The Univ. Tokyo, 2-21-1 Osawa, Mitaka, Tokyo 181-0015, Japan \\ ${ }^{3}$ Dept. Physics, Kyoto Sangyo Univ., Kamo-Motoyama, Kita, Kyoto 603-8555, Japan \\ ${ }^{4}$ National Astronomical Observatory of Japan, 2-21-1 Osawa, Mitaka, Tokyo 181-8588, Japan \\ ${ }^{5}$ Dept. Astronomy, Kyoto Univ., Kitashirakawa-Oiwake-cho, Sakyo, Kyoto 606-8502, Japan \\ ${ }^{6}$ Dept. Physics, Univ. Roma Tor Vergata, Via della Ricerca Scientifica 1, 00133 Rome, Italy
}

\begin{abstract}
We are carrying out near-infrared spectroscopy of Cepheids in the Galactic nuclear disk. The $H$-band spectra taken with SUBARU/IRCS indicate that their kinematics are consistent with the rotation of the nuclear disk.
\end{abstract}

Keywords. stars: variables: Cepheids, Galaxy: bulge, infrared: stars

Cepheid variable stars are useful tracers of the distance scale and stellar populations within the Galaxy. Matsunaga et al. (2011) discovered three Cepheids in the Galactic nuclear bulge (within 200 pc of the Galactic center). With SUBARU IRCS high-resolution spectrograph, we collected $H$-band spectra of the three Cepheids as well as of several additional Cepheids in 2012. The $H$-band spectra of Cepheids show dozens of strong absorption lines (Figure 1). Data analysis is still in progress, but Figure 1 already indicates that their radial velocities are different from each other even considering the Cepheid pulsation effect $( \pm 20 \mathrm{~km} / \mathrm{s})$. The trend of the velocity difference agrees with the rotation of the nuclear disk in that the object (a) in the positive Galactic longitude shows a red shift whereas the others in the positive longitude show blue shifts.

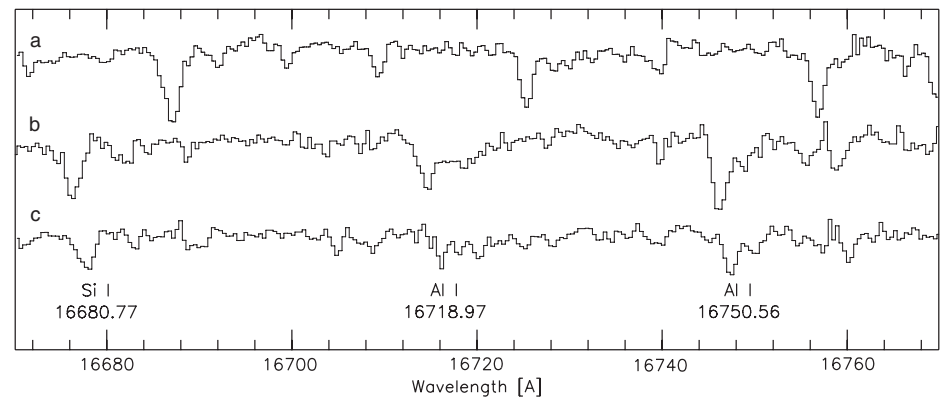

Figure 1. A part of the $H$-band spectra of Cepheids, where some prominent metallic lines are indicated. Differences of the radial velocities are clearly seen.

\section{Reference}

Matsunaga, N., et al. 2011, Nature, 477, 188 